\section{Injectable disease modifying agents in multiple sclerosis: pattern of medication use and clinical effectiveness}

\author{
Elina Järvinen, ${ }^{1}$ Markus Holmberg, 2 \\ Marja-Liisa Sumelahti2 \\ 1Merck Finland, Espoo; 2 School \\ of Medicine, University of Tampere, \\ Finland
}

\section{Abstract}

The objective of this study was to assess long-term use, adherence and efficacy of injectable disease modifying agents (DMAs). Multiple Sclerosis (MS) patients diagnosed during 2002-2010 with early treatment start and at least one year in first choice medication were included in a large university district in Finland. Annualized relapse rates (ARR) during each treatment period were studied, and number of switches by medication evaluated. Use of health care facilities during 2002-2010 was assessed. In the study were included 113 MS patients; 15 (13\%) switched medication. The mean duration of treatment period (128) was 3.8 years. In $77 \%(98 / 128)$ the treatment continued with the first DMA for (mean) 3.8 years, in $19 \%(25 / 128)$ with the second for 3.5 years and in $4 \%(5 / 128)$ with the third for 4.8 years. Mean ARR was 0.26, with 54\% (69/128) of the periods relapse free. Mean ARR during the treatment periods with product switch was 0.41 before, and 0.28 after the switch, showing a trend towards better efficacy with the second DMA. The usage of health care resources remained within the guidelines. Long-term adherence to first choice DMA was observed, and a switch of product within the DMAs showed continuous adherence and efficacy. The efforts to seek a clinically effective and well tolerated agent within the first-line DMAs is warranted, leading to continued adherence and increased clinical effectiveness.

\section{Introduction}

Multiple sclerosis (MS) is a chronic autoimmune disease affecting the central nervous system, and a common cause of physical disability in young adults. ${ }^{1}$ In most cases the disease initially manifests with a relapsing-remitting disease course (RRMS) caused by immune cell infiltration to the central nervous system, which promotes inflammation, demyelination and neurodegeneration. ${ }^{2,3}$ In recent years the treatment options for RRMS have substantially increased. ${ }^{4}$ In Finland, the referral centers for MS patients are central- or university hospitals. Constantly updated national guidelines for MS treatment have instructed treatment practice since $2002 .^{5}$ The disease modifying agents (DMA) in use are interferon beta-1a and interferon-1b, which have been available in Finland since the late 1990 s, and glatiramer acetate (GA) since the early 2000 s. These DMAs are $100 \%$ reimbursed in active MS. It has been shown in several randomized controlled trials and observational studies that these injectable DMAs prevent clinical relapses and disease progression.6-8 They are usually well tolerated, and serious adverse effects are rare. ${ }^{9}$ Over 20 years of experience of the long-term use of injectables has shown that they are effective with good safety profiles.10,11 In case of confirmed disease activity, a switch to other options is justified. New immunosuppressant agents, such as natalizumab and alemtuzumab, however, carry a significant risk for serious adverse effects, 12 and safety precautions may prevent their use. ${ }^{13}$ Here we aim to study the injectable DMA use and adherence, occurrence of medication switch, the clinical effect on relapses, and the usage of health care resources (both for disease activity related and disease monitoring purposes) in a population-based cohort of Finnish MS patients between years 2002 and 2010.

\section{Materials and Methods}

\section{Subjects}

Patients diagnosed by McDonald criteria ${ }^{14}$ with MS (ICD-10 G 35) during 2002-2007 were included from four neurological departments at the central hospitals in Tampere University Hospital District, Finland. Included ambulatory patients had a RRMS disease course with Expanded Disability Status Scale rate less than 5.5 at treatment onset, and had been using the first injectable DMA for a minimum of one year during 2002-2010. Data was collected anonymously via a structured web based questionnaire, which included age at diagnosis, delay from diagnosis to treatment onset, the applied DMAs, and the doses and length of treatment periods, annual relapse rate (ARR), yearly number of visits at neurological centers and MS related hospital days. Statistical significance was calculated using student's t-test or regression analysis when $\mathrm{P}<0.05$. The retrospective examination of identified patient records at hospitals were approved by the Ethics Committee of the University of Tampere Hospital District.
Correspondence: Marja-Liisa Sumelahti, School of Medicine, University of Tampere, $33014 \mathrm{TaY}$, Tampere, Finland.

Tel.: +358.40.587.4149.

E-mail: marja-liisa.sumelahti@uta.fi

Key words: Relapsing remitting multiple sclerosis; DMA; efficacy; long term use; switch.

Acknowledgements: the authors would like to thank Jorma Sormunen and Kenneth Hollmén, for the design of the study, Kati Krühn and Hanne Luoto for preparation of the manuscript and Marketta Kause (Merck Finland) for the study coordination. We thank the following research centers for participation on patient recruitment: Tampere- and Oulu University Central Hospitals, Seinäjoki-, Päijät-Häme-, Kanta-Häme- and Vaasa Central Hospitals, and the Hospital Districts of Helsinki and Uusimaa, and CentralFinland.

Contributions: MLS, collection of material, design of the study, analysis and interpretation of the data, drafting the manuscript; EJ, interpretation of the data, drafting the manuscript; $\mathrm{MH}$, collection of material, analysis and interpretation of the data, drafting the manuscript.

Conflict of interest: Markus Holmberg has no conflict of interest. Elina Järvinen is an employee of Merck Finland, an affiliate of Merck KGaA Darmstadt, Germany. Marja-Liisa Sumelahti has received honoraria for consulting and as a speaker from Novartis, and received grants for congress trips and participation from Novartis, TEVA, Biogen Idec, Bayer, Merck-Serono and Sanofiaventis.

Funding: Funding for this work was provided by Merck Finland, a business of Merck KGaA, Darmstadt, Germany.

Received for publication: 21 March 2016.

Revision received: 13 June 2016.

Accepted for publication: 8 August 2016 .

This work is licensed under a Creative Commons Attribution NonCommercial 4.0 License (CC BYNC 4.0).

(C) Copyright E. Järvinen et al., 2016

Licensee PAGEPress, Italy

Neurology International 2016; 8:6513

doi:10.4081/ni.2016.6513

\section{Results}

A total of 113 RRMS patients (105 women, 8 men) fulfilled the inclusion criteria. Mean age at diagnosis was 36 years (17-54 years). Mean delay from MS diagnosis to first DMA commencement was 8 months (range 0 to 4.41 years). The delay decreased significantly from approximately 11 months in 2002 to approxi- 
mately 3 months in 2007. The DMAs used (Table 1) were interferon beta-1a $30 \mu \mathrm{g}$, i.m. qw, interferon beta-1a $22 \mu$ g, s.c. tiw, interferon beta-1a $44 \mu \mathrm{g}$, s.c. tiw, interferon beta-1b $250 \mu \mathrm{g}$, s.c. eod, and GA $20 \mathrm{mg}$, s.c. daily. Distribution of agents and the mean time of use ranged from 2.9 to 4.6 years. Mean duration of all treatment periods $(\mathrm{n}=128)$ was 3.8 years (1.0-8.5 years). The first choice DMA was used in $77 \%(98 / 128)$ of the treatment periods. In $19 \%$ (25/128) of the periods, the medication had been switched once to a second DMA at mean 2.3 years (0.25-5.14) after diagnosis, and the mean duration of the second treatment period was 3.5 years. In $4 \%$ (5/128) of the periods the patient was treated with a third DMA. The mean time from diagnosis to third medication was 2.6 years, and the mean treatment duration was 4.8 years (0.55.27) (Table 1).

Transitions between the DMAs are shown in Figure 1 . The most frequently (45\%) switched DMA was interferon beta-1a $22 \mu \mathrm{g}(7 / 15)$. In $57 \%$ of these cases (4/7) a transition to a regimen of $44 \mathrm{~g}$ took place. The switch to GA was observed in $60 \%$ of all cases $(9 / 15)$.

ARR was low during the follow-up. The mean ARR was 0.26 [0-2.31, standard deviation (SD) 0.42 ]. In $54 \%(69 / 128)$ of the treatment periods the patients did not experience any relapses. Total of $15(13 \%, 15 / 113)$ subjects switched medication (Figure 1). In this subgroup the mean ARR decreased non-significantly from $0.41(0-1.1)$ to $0.28(0-2.1)$ after the switch. The mean time on treatment increased from 2.5 years (1.0-4.7) to 3.7 years (1.0-6.2) after the conversion.

The yearly mean number of control visits with a neurologist in the whole cohort was 1.3 $(0-3.8)$, and with a MS nurse $1.0(0-3.8)$. The mean yearly number of additional visits with a neurologist was $0.2(0-2.9)$. Mean number of hospital days due to MS was $0.6(0-13.1)$ per year.

\section{Discussion and Conclusions}

In this study we evaluated the long-term adherence and efficacy of DMAs in a relapsingremitting MS patient population by studying the length of the treatment periods and the annual relapse rate related to medication switch. In this long-term clinical setting also the usage of health care resources was investigated.

The selection of cases was limited to patients who are expected to tolerate the chosen medication for a long period. The population represented an early treatment group, as in most of the cases treatment started within a year from diagnosis, 15 and cases were expected to show disease activity. Side effects or development of neutralizing antibodies, which appear mainly during the first years of treatment, may lead to treatment switch. Therefore, for the purposes of this study, we only included patients who had stayed on a DMA for at least one year and no neutralizing antibodies were detected during the first year.

The clinical effectiveness was evaluated by ARR, and the proportion of relapse-free patients. In all treatment periods a low ARR was observed, pointing at continuous disease activity in about half of the patients. During the follow-up a switch of treatment took place in $22 \%$ of treatment periods, mostly from first to second treatment option in $19 \%$ of the cases. In most of these cases the change to a higher dose of interferon beta-1a or switch to GA was

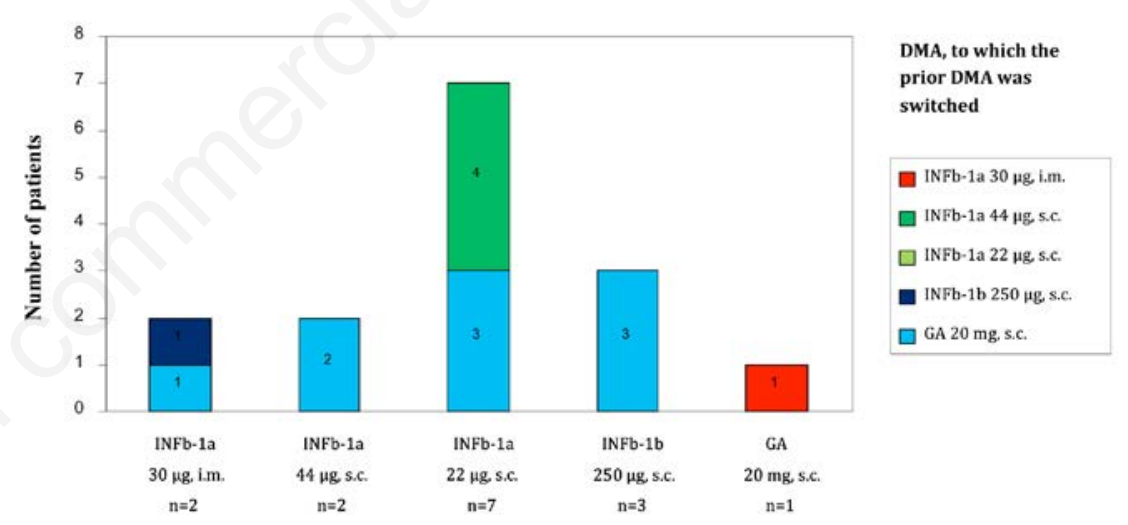

Prior DMA, which was switched to another DMA

Figure 1. Multiple Sclerosis patients with observed switches and injectable disease modifying agents transitions (treatment duration $\geq 1$ year). observed. We did not characterize the causes. It is likely that those who switched medication represent cases with treatment inefficacy and tolerability issues.6,8 Long-term side effects in DMA use are frequent. Irrespective of the cause of switch, the population that switched to a second DMA (13\% of the patients) a higher initial ARR was observed, but after the switch, these patients reached a lower mean ARR similar to the ARR observed in the whole population, pointing at a good response to the second DMA medication. This result supports the rationale for the switch. At the time of the study choices for more active treatments, such as natalizumab, were limited.16 Interestingly, in the clinical trial for alemtuzumab, the reduction in risk of relapses with alemtuzumab was lower in patients who had not been previously treated with interferon beta-1a $44 \mu \mathrm{g}$, s.c. tiw, ${ }^{17}$ suggesting that patients who do not respond to another interferon or GA, may benefit from an initial switch to interferon beta-la $44 \mu$ g, s.c. tiw, before embarking on the more risky option of alemtuzumab.

We observed that the usage of health care resources was moderate i.e. the number of neurological clinic visits was in line with the recommendation for regular yearly visits, ${ }^{5}$ and the mean number of hospital days was less than one day per year, supporting the efficacy and benefits of continuous and long-term use

Table 1. Usage of disease modifying agents (DMAs) during the treatment periods from 2002 to 2010, distribution by consecutive treatment period, duration of treatment period by DMA, and treatment time in the treatment periods.

\begin{tabular}{|c|c|c|c|c|c|c|}
\hline \multirow[t]{2}{*}{ Disease modifying agent } & \multirow[t]{2}{*}{$\begin{array}{c}\text { Treatment } \\
\text { periods n. (\%) }\end{array}$} & \multirow[t]{2}{*}{$\begin{array}{l}\text { Mean time on } \\
\text { treatment (years) }\end{array}$} & \multirow[t]{2}{*}{$\begin{array}{l}\text { Treatment time } \\
\text { in periods (years) }\end{array}$} & \multicolumn{3}{|c|}{$\begin{array}{c}\text { Distribution in } \\
\text { treatment periods, } \mathrm{n}(\%)\end{array}$} \\
\hline & & & & 1st DMA & 2nd DMA & 3rd DMA \\
\hline Interferon beta-la $30 \mu \mathrm{g}$, i.m. qw & $17(13)$ & 4.1 & $1.0-8.5$ & $15(88)$ & $1(6)$ & $1(6)$ \\
\hline Interferon beta-la $44 \mu$ g s.c. tiw & $20(16)$ & 2.9 & $1.1-4.9$ & $11(55)$ & $8(40)$ & $1(5)$ \\
\hline Interferon beta-la $22 \mu$ g s.c. tiw & $41(32)$ & 4 & $1.0-7.8$ & $39(95)$ & $2(5)$ & $0(0)$ \\
\hline Interferon beta-1b $250 \mu$ g s.c. eod & $30(23)$ & 3.6 & $1.3-7.8$ & $93(28)$ & $2(7)$ & $0(0)$ \\
\hline Glatiramer acetate $20 \mathrm{mg}$ s.c. eod & $20(16)$ & 4.6 & $2.4-7.8$ & $5(25)$ & $12(60)$ & $3(15)$ \\
\hline Total & $128(100)$ & 3.8 & $1.0-8.5$ & $98(77)$ & $25(19)$ & $5(4)$ \\
\hline
\end{tabular}


of DMAs. A limitation of our study was the low number of subjects in this study, 113 patients and 128 treatment periods, and undermines the significance of the results.

In this study the follow-up time was at least one year to ensure maximal medication effectiveness. Long-term follow-up data show that higher and longer cumulative exposure to interferon beta-1a is associated with better clinical outcomes in relapsing-remitting MS, 11 and earlier and longer exposure to interferon beta treatment improves survival of the patients with MS.10 Our results support the importance of an early intervention and an effective and well tolerated long-term treatment. The extending selection of more powerful MS treatments persuades neurologists and patients with improved effectiveness and compliance, but at the same time managing the risks of adverse events has become more demanding. 16

In conclusion, we observed a positive response to a long-term treatment with DMAs, manifesting in reduced ARR among patients who switched between injectable DMAs. Our results suggest that if the treatment effect is not desirable or the patient suffers from side effects, but the disease shows non-aggressive activity, a switch within the injectable DMA medication group can be justified.

\section{References}

1. Compston A, Coles A. Multiple sclerosis. Lancet 2002;359:1221-31.

2. Dendrou CA, Fugger L, Friese MA.
Immunopathology of multiple sclerosis. Nat Rev Immunol 2015;15:545-58.

3. Lublin FD. New multiple sclerosis phenotypic classification. Eur Neurol 2014;72:15.

4. Wiendl H, Meuth SG. Pharmacological approaches to delaying disability progression in patients with multiple sclerosis. Drugs 2015;75:947-77.

5. Suomen Neurologinen Yhdistys ry. [Diagnostics and drug therapy of multiple sclerosis]. Duodecim 2002;118:1411-23. [Article in Finnish]

6. Randomised double-blind placebo-controlled study of interferon beta-1a in relapsing/remitting multiple sclerosis. PRISMS (Prevention of Relapses and Disability by Interferon beta-1a Subcutaneously in Multiple Sclerosis) Study Group. Lancet 1998;352:1498-504.

7. Panitch H, Goodin DS, Francis G, et al. Randomized, comparative study of interferon beta-1a treatment regimens in MS: The EVIDENCE Trial. Neurology 2002;59:1496-506.

8. Mikol DD, Barkhof F, Chang $\mathrm{P}$, et al. Comparison of subcutaneous interferon beta-1a with glatiramer acetate in patients with relapsing multiple sclerosis (the REbif vs Glatiramer Acetate in Relapsing MS Disease [REGARD] study): a multicentre, randomised, parallel, open-label trial. Lancet Neurol 2008;7:903-14.

9. O'Connor PW, Oh J. Disease-modifying agents in multiple sclerosis. Handb Clin Neurol 2014;122:465-501.

10. Goodin D, Ebers G, Cutter G, et al. Cause of death in MS: long-term follow-up of a ran- domised cohort, 21 years after the start of the pivotal IFN-1b study. BMJ Open $2012 ; 2$.

11. Kappos L, Kuhle J, Multanen J, et al. Factors influencing long-term outcomes in relapsing-remitting multiple sclerosis: PRISMS-15. J Neurol Neurosurg Psychiatry 2015;86:1202-7.

12. Warnke C, Olsson T, Hartung HP. PML: the dark side of immunotherapy in multiple sclerosis. Trends Pharmacol Sci 2015;36:799-801.

13. Monaco MC, Major EO. Immune system involvement in the pathogenesis of $\mathrm{JC}$ virus induced PML: what is learned from studies of patients with underlying diseases and therapies as risk factors. Front Immunol 2015;28:159.

14. McDonald I, Compston A, Edan G, et al. Recommended diagnostic criteria for multiple sclerosis: guidelines from the panel on the diagnosis of multiple sclerosis. Ann Neurol 2001;50:121-7.

15. Reder AT, Ebers GC, Traboulsee A, et al. Cross-sectional study assessing long-term safety of interferon-beta-1b for relapsingremitting MS. Neurology 2010;74:1877-85.

16. Straus Farber R, Harel A, Lublin F. Novel agents for relapsing forms of multiple sclerosis. Annu Rev Med 2015;17.

17. Coles AJ, Twyman CL, Arnold DL, et al. Alemtuzumab for patients with relapsing multiple sclerosis after disease-modifying therapy: a randomised controlled phase 3 trial. Lancet 2012;380:1829-39. 